\title{
The cubics which are differences of two conjugates of an algebraic integer
}

\author{
par TOUFIK ZAIMI
}

\begin{abstract}
RÉSUMÉ. On montre qu'un entier algébrique cubique sur un corps de nombres $K$, de trace nulle est la différence de deux conjugués sur $K$ d'un entier algébrique. On prouve aussi que si $N$ est une extension cubique normale du corps des rationnels, alors tout entier de $N$ de trace zéro est la différence de deux conjugués d'un entier de $N$ si et seulement si la valuation 3 -adique du discriminant de $N$ est différente de 4 .
\end{abstract}

ABStRaCt. We show that a cubic algebraic integer over a number field $K$, with zero trace is a difference of two conjugates over $K$ of an algebraic integer. We also prove that if $N$ is a normal cubic extension of the field of rational numbers, then every integer of $N$ with zero trace is a difference of two conjugates of an integer of $N$ if and only if the 3-adic valuation of the discriminant of $N$ is not 4 .

\section{Introduction}

Let $K$ be a number field, $\beta$ an algebraic number with conjugates $\beta_{1}=$ $\beta, \beta_{2}, \ldots, \beta_{d}$ over $K$ and $L=K\left(\beta_{1}, \beta_{2}, \ldots, \beta_{d}\right)$ the normal closure of the extension $K(\beta) / K$. In [2], Dubickas and Smyth have shown that $\beta$ can be written $\beta=\alpha-\alpha^{\prime}$, where $\alpha$ and $\alpha^{\prime}$ are conjugates over $K$ of an algebraic number, if and only if there is an element $\sigma$ of the Galois group $G(L / K)$ of the extension $L / K$, of order $n$ such that $\sum_{0 \leq i \leq n-1} \sigma^{i}(\beta)=0$. In this case $\beta=\alpha-\sigma(\alpha)$, where $\alpha=\sum_{0 \leq i \leq n-1}(n-i-1) \sigma^{i}(\beta) / n$ is an element of $L$ and the trace of $\beta$ for the extension $K(\beta) / K$, namely $\operatorname{Tr}_{K(\beta) / K}(\beta)=$ $\beta_{1}+\beta_{2}+\ldots+\beta_{d}$, is 0 . Furthermore, the condition on the trace of $\beta$ to be 0 is also sufficient to express $\beta=\alpha-\alpha^{\prime}$ with some $\alpha$ and $\alpha^{\prime}$ conjugate over $K$ of an algebraic number, when the extension $K(\beta) / K$ is normal (i. e. when $L=K(\beta)$ ) and its Galois group is cyclic (in this case we say that the extension $K(\beta) / K$ is cyclic) or when $d \leq 3$.

Manuscrit reçu le 20 décembre 2003. 
Let $D$ be a positive rational integer and $\mathcal{P}(D)$ the proposition : For any number field $K$ and for any algebraic integer $\beta$ of degree $\leq D$ over $K$, if $\beta$ is a difference of two conjugates over $K$ of an algebraic number, then $\beta$ is a difference of two conjugates over $K$ of an algebraic integer. In [1], Smyth asked whether $\mathcal{P}(D)$ is true for all values of $D$. It is clear that if $\operatorname{Tr}_{K(\beta) / K}(\beta)=0$ and $\beta \in \mathbb{Z}_{K}$, where $\mathbb{Z}_{K}$ is the ring of integers of $K$, then $\beta=0=0-0$ and $\mathcal{P}(1)$ is true. For a quadratic extension $K(\beta) / K$, Dubickas showed that if $\operatorname{Tr}_{K(\beta) / K}(\beta)=0$, then $\beta$ is a difference of two conjugates over $K$ of an algebraic integer of degree $\leq 2$ over $K(\beta)$ [1]. Hence, $\mathcal{P}(2)$ is true. In fact, Dubickas proved that if the minimal polynomial of the algebraic integer $\beta$ over $K$, say $\operatorname{Irr}(\beta, K)$, is of the form $P\left(x^{m}\right)$, where $P \in \mathbb{Z}_{K}[x]$ and $m$ is a rational integer greater than 1 , then $\beta$ is a difference of two conjugates over $K$ of an algebraic integer.

Consider now the assertion $\mathcal{P}_{c}(D)$ : For any number field $K$ and for any algebraic integer $\beta$ of degree $\leq D$ over $K$ such that the extension $K(\beta) / K$ is cyclic, if $\operatorname{Tr}_{K(\beta) / K}(\beta)=0$, then $\beta$ is a difference of two conjugates over $K$ of an algebraic integer.

The first aim of this note is to prove :

Theorem 1. The assertions $\mathcal{P}(D)$ and $\mathcal{P}_{c}(D)$ are equivalent, and $\mathcal{P}(3)$ is true.

Let $\mathbb{Q}$ be the field of rational numbers. In [5], the author showed that if the extension $N / \mathbb{Q}$ is normal with prime degree, then every integer of $N$ with zero trace is a difference of two conjugates of an integer of $N$ if and only if $\operatorname{Tr}_{N / \mathbb{Q}}\left(\mathbb{Z}_{N}\right)=\mathbb{Z}_{\mathbb{Q}}$. It easy to check that if $N=\mathbb{Q}(\sqrt{m})$ is a quadratic field ( $m$ is a squarefree rational integer), then $\operatorname{Tr}_{N / \mathbb{Q}}\left(\mathbb{Z}_{N}\right)=\mathbb{Z}_{\mathbb{Q}}$ if and only if $m \equiv 1[4]$. For the cubic fields we have :

Theorem 2. Let $N$ be a normal cubic extension of $\mathbb{Q}$. Then, every integer of $N$ with zero trace is a difference of two conjugates of an integer of $N$ if and only if the 3-adic valuation of the discriminant of $N$ is not 4 .

\section{Proof of Theorem 1}

First we prove that the propositions $\mathcal{P}(D)$ and $\mathcal{P}_{c}(D)$ are equivalent. It is clear that $\mathcal{P}(D)$ implies $\mathcal{P}_{c}(D)$, since by Hilbert's Theorem 90 [3] the condition $\operatorname{Tr}_{K(\beta) / K}(\beta)=0$ is sufficient to express $\beta=\alpha-\alpha^{\prime}$ with some $\alpha$ and $\alpha^{\prime}$ conjugate over $K$ of an algebraic number. Conversely, let $\beta$ be an algebraic integer of degree $\leq D$ over $K$ and which is a difference of two conjugates over $K$ of an algebraic number. By the above result of Dubickas and Smyth, and with the same notation, there is an element $\sigma \in G(L / K)$, of order $n$ such that $\sum_{0 \leq i \leq n-1} \sigma^{i}(\beta)=0$. Let $\langle\sigma\rangle$ be the cyclic subgroup of $G(L / K)$ generated by $\sigma$ and $L^{<\sigma>}=\{x \in L, \sigma(x)=x\}$ the fixed field of $\langle\sigma\rangle$. Then, $K \subset L^{\langle\sigma\rangle} \subset L^{<\sigma\rangle}(\beta) \subset L$, the degree of $\beta$ over $L^{<\sigma\rangle}$ is 
$\leq D$ and by Artin's theorem [3], the Galois group of the normal extension $L / L^{<\sigma\rangle}$ is $\langle\sigma\rangle$. Hence, the extensions $L / L^{<\sigma>}$ and $L^{<\sigma>}(\beta) / L^{<\sigma\rangle}$ are cyclic since their Galois groups are respectively $\langle\sigma\rangle$ and a factor group of $\langle\sigma\rangle$. Furthermore, the restrictions to the field $L^{<\sigma>}(\beta)$ of the elements of the group $\langle\sigma\rangle$ belong to the Galois group of $L^{<\sigma>}(\beta) / L^{<\sigma\rangle}$ and each element of $G\left(L^{<\sigma>}(\beta) / L^{<\sigma>}\right)$ is a restriction of exactly $d$ elements of the group $\langle\sigma\rangle$, where $d$ is the degree of $L / L^{<\sigma\rangle}(\beta)$. It follows that

$$
d \operatorname{Tr}_{L^{<\sigma>}(\beta) / L<\sigma>}(\beta)=\operatorname{Tr}_{L / L<\sigma>}(\beta)=\sum_{0 \leq i \leq n-1} \sigma^{i}(\beta)=0,
$$

and $\beta$ is a difference of two conjugates over $L^{<\sigma>}$ of an algebraic number. Assume now that $\mathcal{P}_{c}(D)$ is true. Then, $\beta$ is difference of two conjugates over $L^{<\sigma\rangle}$, and a fortiori over $K$, of an algebraic integer and so $\mathcal{P}(D)$ is true.

To prove that $\mathcal{P}(3)$ is true, it is sufficient to show that if $\beta$ a cubic algebraic integer over a number field $K$ with $\operatorname{Tr}_{K(\beta) / K}(\beta)=0$ and such that the extension $K(\beta) / K$ is cyclic, then $\beta$ is a difference of two conjugates of an algebraic integer, since $\mathcal{P}(2)$ is true and the assertions $\mathcal{P}(3)$ and $\mathcal{P}_{c}(3)$ are equivalent. Let

$$
\operatorname{Irr}(\beta, K)=x^{3}+p x+q,
$$

and let $\sigma$ be a generator of $G(K(\beta) / K)$. Then, $p=\operatorname{Tr}_{K(\beta) / K}(\beta \sigma(\beta))$ and the discriminant $\operatorname{disc}(\beta)$ of the polynomial $\operatorname{Irr}(\beta, K)$ satisfies

$$
\operatorname{disc}(\beta)=-4 p^{3}-3^{3} q^{2}=\delta^{2}
$$

where $\delta=\left(\beta-\sigma^{2} \beta\right)(\sigma \beta-\beta)\left(\sigma^{2} \beta-\sigma \beta\right) \in \mathbb{Z}_{K}$. Set $\gamma=\beta-\sigma^{2}(\beta)$. Then, $\gamma$ is of degree 3 over $K$ and

$$
\operatorname{Irr}(\gamma, K)=x^{3}+3 p x-\delta .
$$

As the polynomial $-27 t+x^{3}+3 p x-26 \delta$ is irreducible in the ring $K(\beta)[t, x]$, by Hilbert's irreducibility theorem [4], there is a rational integer $s$ such that the polynomial $x^{3}+3 p x-(26 \delta+27 s)$ is irreducible in $K(\beta)[x]$. Hence, if $\theta^{3}+3 p \theta-(26 \delta+27 s)=0$, then

$$
\operatorname{Irr}(\theta, K(\beta))=x^{3}+3 p x-(26 \delta+27 s)=\operatorname{Irr}(\theta, K),
$$

since $\operatorname{Irr}(\theta, K(\beta)) \in K[x]$. Set $\alpha=\frac{\gamma}{3}+\frac{\theta}{3}$. Then, $\frac{\sigma(\gamma)}{3}+\frac{\theta}{3}$ is a conjugate of $\alpha$ over $K(\beta)$ (and a fortiori over $K$ ) and

$$
\beta=\frac{\gamma}{3}+\frac{\theta}{3}-\left(\frac{\sigma(\gamma)}{3}+\frac{\theta}{3}\right) .
$$

From the relations $\left(\frac{\theta}{3}\right)^{3}+\frac{p}{3}\left(\frac{\theta}{3}\right)-\frac{26 \delta+27 s}{27}=0$ and $\left(\frac{\gamma}{3}\right)^{3}+\frac{p}{3}\left(\frac{\gamma}{3}\right)=\frac{\delta}{27}$, we obtain that $\alpha$ is a root of the polynomial

$$
x^{3}-\gamma x^{2}+\left(\frac{\gamma^{2}+p}{3}\right) x-(\delta+s) \in K(\beta)[X]
$$


and $\alpha$ is an algebraic integer (of degree $\leq 3$ over $K(\beta)$ ) provided $\frac{\gamma^{2}+p}{3} \in$ $\mathbb{Z}_{K(\beta)}$. A short computation shows that from the relation $\gamma\left(\gamma^{2}+3 p\right)=\delta$, we have $\operatorname{Irr}\left(\frac{\gamma^{2}}{3}, K\right)=x^{3}+2 p x^{2}+p^{2} x-\frac{\operatorname{disc}(\beta)}{27}$ and $\frac{\gamma^{2}+p}{3}$ is a root of the polynomial $x^{3}+p x^{2}+q^{2}$ whose coefficients are integers of $K$.

Remark 1. It follows from the proof of Theorem 1 , that if $\beta$ is a cubic algebraic integer over a number field $K$ with zero trace, then $\beta$ is a difference of two conjugates over $K$ of an algebraic integer of degree $\leq 3$ over $K(\beta)$. The following example shows that the constant 3 in the last sentence is the best possible. Set $K=\mathbb{Q}$ and $\operatorname{Irr}(\beta, \mathbb{Q})=x^{3}-3 x-1$. Then, $\operatorname{disc}(\beta)=3^{4}$ and the extension $\mathbb{Q}(\beta) / \mathbb{Q}$ is normal, since $\beta^{2}-2$ is also a root of $\operatorname{Irr}(\beta, \mathbb{Q})$. By Theorem 3 of [5], $\beta$ is not a difference of two conjugates of an integer of $\mathbb{Q}(\beta)$ (the 3 -adic valuation of $\operatorname{disc}(\beta)$ is 4 ) and if $\beta=\alpha-\alpha^{\prime}$, where $\alpha$ is an algebraic integer of degree 2 over $\mathbb{Q}(\beta)$ and $\alpha^{\prime}$ is a conjugate of $\alpha$ over $\mathbb{Q}(\beta)$, then there exists an element $\tau$ of the group $G(\mathbb{Q}(\beta, \alpha) / \mathbb{Q}(\beta))$ such that $\tau(\beta)=\beta, \tau(\alpha)=\alpha^{\prime}, \tau\left(\alpha^{\prime}\right)=\alpha$ and $\beta=\tau\left(\alpha-\alpha^{\prime}\right)=\alpha^{\prime}-\alpha=-\beta$.

Remark 2. With the notation of the proof of Theorem 1 (the second part) we have: Let $\beta$ be a cubic algebraic integer over $K$ with zero trace and such that the extension $K(\beta) / K$ is cyclic. Then, $\beta$ is a difference of two conjugates of an integer of $K(\beta)$, if and only if there exists $a \in \mathbb{Z}_{K}$ such that the two numbers $\frac{a^{2}+p}{3}$ and $\frac{a^{3}+3 p a+\delta}{27}$ are integers of $K$. Indeed, suppose that $\beta=\alpha-\sigma(\alpha)$, where $\alpha \in \mathbb{Z}_{K(\beta)}$ (if $\beta=\alpha-\sigma^{2}(\alpha)$, then $\beta=\alpha+\sigma(\alpha)-\sigma(\alpha+\sigma(\alpha)))$. Then, $\alpha-\sigma(\alpha)=\frac{\gamma}{3}-\sigma\left(\frac{\gamma}{3}\right), \alpha-\frac{\gamma}{3}=\sigma\left(\alpha-\frac{\gamma}{3}\right)$, $\alpha-\frac{\gamma}{3} \in K$ and there exists an integer $a$ of $K$ such that $3 \alpha-\gamma=a$. Hence, $\frac{\gamma+a}{3}=\alpha \in \mathbb{Z}_{K(\beta)}, \operatorname{Irr}\left(\frac{\gamma+a}{3}, K\right)=x^{3}-a x^{2}+\frac{a^{2}+p}{3} x-\frac{a^{3}+3 p a+\delta}{27} \in \mathbb{Z}_{K}[X]$ and so the numbers $\frac{a^{2}+p}{3}$ and $\frac{a^{3}+3 p a+\delta}{27}$ are integers of $K$. The converse is trivial, since $\beta=\frac{\gamma}{3}-\sigma\left(\frac{\gamma}{3}\right)=\frac{\gamma+a}{3}-\sigma\left(\frac{\gamma+a}{3}\right)$ for all integers $a$ of $K$. It follows in particular when $\frac{\operatorname{disc}(\beta)}{3^{6}} \in \mathbb{Z}_{K}$, that $\beta$ is a difference of two conjugates of an integer of $K(\beta)(a=0)$. Note finally that for the case where $K=\mathbb{Q}$ a more explicit condition was obtained in [5].

\section{Proof of Theorem 2}

With the notation of the proof of Theorem 1 (the second part) and $K=\mathbb{Q}$, let $N$ be a cubic normal extension of $\mathbb{Q}$ with discriminant $\Delta$ and let $v$ be the 3 -adic valuation. Suppose that every non-zero integer $\beta$ of $N$ with zero trace is a difference of two conjugates of an integer of $N$. Then, $N=\mathbb{Q}(\beta)$ and by Theorem 3 of $[5], v(\operatorname{disc}(\beta)) \neq 4$. Assume also $v(\Delta)=4$. Then, $v(\operatorname{disc}(\beta))>4$ and hence $v(\operatorname{disc}(\beta)) \geq 6$, since $\frac{\operatorname{disc}(\beta)}{\Delta} \in \mathbb{Z}_{\mathbb{Q}}$ and $\operatorname{disc}(\beta)$ is a square of a rational integer. It follows that $\frac{\gamma}{3}$ is an algebraic 
integer, since its minimal polynomial over $\mathbb{Q}$ is $x^{3}+\frac{p}{3} x-\frac{\delta}{27} \in \mathbb{Z}_{\mathbb{Q}}[X]$ and $\beta$ can be written $\beta=\alpha-\sigma(\alpha)$, where $\alpha=\frac{\gamma}{3}$ is an integer of $N$ with zero trace. Thus, $v(\operatorname{disc}(\alpha)) \geq 6$ and there is an integer $\eta$ of $N$ with zero trace, such that $\alpha=\eta-\sigma(\eta)$. It follows that $\beta=\eta-\sigma(\eta)-\sigma(\eta-\sigma(\eta))=$ $\eta-2 \sigma(\eta)+\sigma^{2}(\eta)=-3 \sigma(\eta)$ and $\frac{\beta}{3}$ is also an integer of $N$ with zero trace. The last relation leads to a contradiction since in this case $\frac{\beta}{3^{n}} \in \mathbb{Z}_{N}$ for all positive rational integers $n$. Conversely, suppose $v(\Delta) \neq 4$. Assume also that there exists an integer $\beta$ of $N$ with zero trace which is not a difference of two conjugates of an integer of $N$. Then, $N=\mathbb{Q}(\beta)$ and by Theorem 1 of [5], we have $\operatorname{Tr}_{N / \mathbb{Q}}\left(\mathbb{Z}_{N}\right)=3 \mathbb{Z}$, since $\operatorname{Tr}_{N / \mathbb{Q}}(1)=3$ and $T r_{N / \mathbb{Q}}\left(\mathbb{Z}_{N}\right)$ is an ideal of $\mathbb{Z}$. If $\left\{e_{1}, e_{2}, e_{3}\right\}$ is an integral basis of $N$, then from the relation $\Delta=\operatorname{det}\left(\operatorname{Tr}\left(e_{i} e_{j}\right)\right)$, we obtain $v(\Delta) \geq 3$ and hence $v(\Delta) \geq 6$, since $\Delta$ is a square of a rational integer. The last inequality leads to a contradiction as in this case we have $v(\operatorname{disc}(\beta)) \geq 6$ and $\beta=\frac{\gamma}{3}-\sigma\left(\frac{\gamma}{3}\right)$ where $\frac{\gamma}{3} \in \mathbb{Z}_{N}$.

This work is partially supported by the research center ( $N^{o}$ Math/1419/20).

\section{References}

[1] A. DUBICKAS, On numbers which are differences of two conjugates of an algebraic integer. Bull. Austral. Math. Soc. 65 (2002), 439-447.

[2] A. Dubickas, C. J. Sмyтh, Variations on the theme of Hilbert's Theorem 90. Glasg. Math. J. 44 (2002), 435-441.

[3] S. LANG, Algebra. Addison-Wesley Publishing, Reading Mass. 1965.

[4] A. Schinzel, Selected Topics on polynomials. University of Michigan. Ann Arbor, 1982.

[5] T. ZAIMI, On numbers which are differences of two conjugates over $\mathbb{Q}$ of an algebraic integer. Bull. Austral. Math. Soc. 68 (2003), 233-242.

Toufik ZAIMI

King Saud University

Dept. of Mathematics P. O. Box 2455

Riyadh 11451, Saudi Arabia

E-mail : zaimitou@ksu.edu.sa 